\title{
Rotational dynamics of magnetic particles in suspensions
}

\author{
Claudio Scherer, ${ }^{1,2}$ and Hans-Georg Matuttis ${ }^{1}$ \\ ${ }^{1}$ Institute for Computer Applications 1, University of Stuttgart 70569 Stuttgart, Germany \\ ${ }^{2}$ Institute of Physics, Federal University of Rio Grande do Sul 91501-970 Porto Alegre, Rio Grande do Sul, Brazil
}

(Received 12 November 1999; revised manuscript received 31 August 2000; published 19 December 2000)

\begin{abstract}
An approach for the rotational dynamics of magnetic particles and their magnetic moments, in fluid suspensions, is developed. A possible application is to magnetic resonance in ferrofluids. Based on a generalized Lagrangian formulation for the equations of motion of the particle, we introduce its interaction with the solvent fluid via dissipative and random noise torques, as well as the interaction between the particle and its magnetic moment, treated as an independent physical entity and characterized by three generalized coordinates: its two polar angles and its modulus. In the appropriate limits, it reduces to the cases of superparamagnetic particles or nonsuperparamagnetic (blocked magnetic moments) particles. It is also indicated how the dynamic complex susceptibility may be calculated from the equations of motion, and as an example the effect of the particles inertia on the susceptibility is numerically evaluated for some arbitrary values of the parameters.
\end{abstract}

DOI: 10.1103/PhysRevE.63.011504

PACS number(s): 05.20.Jj, 75.50.Mm

\section{INTRODUCTION}

Considerable interest has been shown, in recent years, in the dynamics of the magnetization of ferrofluids in the presence of applied magnetic fields, and in the corresponding complex magnetic susceptibility. Just to give a few examples of recently published work on the field, we mention theoretical works by Raikher and Rusakov [1], Coffey and Kalmykov [2], and Shliomis and Stepanov [3], experimental works by Morais et al. [4], Fannin et al. [5], Vincent et al. [6], and Upadhyay, Srinivas, and Mehta [7], and an experimental-theoretical paper by Fannin, Kinsella, and Charles [8]. Certainly, this increased interest in a better understanding of the behavior of these materials is related to their renewed technological importance, with various new applications [9].

The usual theoretical approach to calculate the dynamic susceptibility is based on Gilber's [10] or Landau and Lifshitz' [11] equations (which are equivalent) for the dynamics of the magnetic moment, with the addition of noise, following the pioneering work of Brown [12]. Several authors used these equations of motion to calculate relaxation times, and the susceptibility is then borrowed from Debye's theory [13].

Two distinct rotational relaxation mechanisms may coexist in ferrofluids: the Néel relaxation, by which the magnetic moment moves with respect to the mechanical particle; and the Brownian, or Debye relaxation, corresponding to the particle's rotation inside the fluid. In most experimental situations one of these mechanisms is dominant, and this may be the reason why up to now, as far as we know, there has been no satisfactory theory, sufficiently general to be applied for all situations, from the pure Néel motion to the pure Brownian relaxation, passing by all possible combinations of those mechanisms. In this respect the model of "two spheres," by Fannin and Coffey [14], should be mentioned as a first effort. The noninertial limit, i.e., when the contribution of the moments of inertia of the particle to the equations of motion is negligible in comparison to the other forces involved, was treated by Shliomis and Stepanov [3], where they introduced the egg model. There they compared the magnetic particle with an egg, the yolk corresponding to the magnetic moment, and showed that in the noninertial limit and for weak applied field the equations of motion decouple, thus allowing one to account simultaneously for the combined motion of the magnetic moment and the particle.

The purpose of the present paper is to present a general set of equations of motion for the combined system of a magnetic moment plus a mechanical particle, inside a fluid. The main limitation of our approach is that we deal only with axially symmetric particles, with easy axes of magnetization parallel to the symmetry axis. However, the magnetic moment is allowed to rotate inside the particle, as well as to have an oscillating modulus, and the particle is allowed to rotate with respect to the solvent, which is immobile with respect to the laboratory. The suspension is considered sufficiently dilute for the particle-particle interaction to be negligible, so that we deal only with single particle dynamics.

In contradistinction to most existing theories, to our knowledge, our approach includes the particle's moment of inertia in the equations of motion. To neglect inertia may be a good approximation for most ferrofluids, because of the smallness of the particles, but we are presenting a theory which intends to be sufficiently general to include nonstable suspensions, for which the particles may be considerably larger. In the case of superparamagnetic particles, an aspect which distinguishes our theory from the usual approaches is that the rotation of the potential gradient on the magnetic moment, accompanying the Brownian rotation of the particle, is taken into account.

In Sec. II we write the equations of the rotational motion of an axially symmetric particle inside a fluid (Langevin-type equations), based on the generalized Euler-Lagrange equations. In Sec. III we obtain, from the equations of Sec. II, in a convenient limit, the equations of motion for the magnetic moment $\boldsymbol{\mu}$, which reduce, in the case of constant modulus of $\boldsymbol{\mu}$, to the Gilbert's equation. In Sec. IV we arrive at a set of six coupled equations, for the six degrees of freedom, the three Euler angles of the particle's rotations, and the two polar angles of $\boldsymbol{\mu}$ and its modulus. In Sec. V we indicate, briefly, how to calculate, by numerical simulation of the 
equations of motion, the dynamic magnetic susceptibility, a procedure which was more carefully explained in a previous paper [15]. Some less general situations are considered in Sec. VI as particular cases, and related to previous works made by several authors.

\section{ROTATIONAL DYNAMICS OF A PARTICLE IN A FLUID}

Consider a particle of axially symmetric shape in suspension in a fluid. The principal moments of inertia will be denoted by $I_{1}=I_{2}$ and $I_{3}$. Disregarding translational degrees of freedom, its Lagrangian may be written in terms of the Euler angles $\theta, \phi$, and $\psi$ (in the notation of Goldstein [16]), taken as generalized coordinates, as

$$
L=\frac{I_{1}}{2}\left(\dot{\theta}^{2}+\dot{\phi}^{2} \sin ^{2} \theta\right)+\frac{I_{3}}{2}(\dot{\psi}+\dot{\phi} \cos \theta)^{2}-V(\theta, \phi),
$$

where $V(\theta, \phi)$ is some orientation-dependent potential. It cannot depend on $\psi$ because of the axial symmetry of the particle.

The interaction forces (torques) between the particle and the fluid are of dissipative and noise types. Therefore, they are not included in the Lagrangian; instead, we use "generalized Euler-Lagrange equations," with the corresponding torques, represented by $Q_{i}$, at the right hand side,

$$
\frac{d}{d t} \frac{\partial L}{\partial \dot{q}_{i}}-\frac{\partial L}{\partial q_{i}}=Q_{i}
$$

where $q_{i}=\theta, \phi$, or $\psi$.

We write the nonconservative torques $Q_{i}$ as sums of dissipative and noise terms, in the form

$$
Q_{i}=-\frac{\partial \mathcal{F}}{\partial \dot{q}_{i}}+\Gamma_{i}(t)
$$

where $\mathcal{F}$ is the following Rayleigh dissipation function [16],

$$
\mathcal{F}=\frac{1}{2} \lambda\left[\left(\dot{\theta}^{2}+\dot{\phi}^{2} \sin ^{2} \theta\right)\right]+\frac{1}{2} \lambda^{\prime}(\dot{\psi}+\dot{\phi} \cos \theta)^{2}
$$

and $\Gamma_{i}(t)$ are the noise torques. The dissipation constants $\lambda$ and $\lambda^{\prime}$ may be different because $\lambda^{\prime}$ is associated with the particle rotation around the symmetry axis, while $\lambda$ is associated with the rotations perpendicular to it. Substituting Eqs. (1), (3), and (4) into Eq. (2) we obtain the following system of equations for the particle's rotation:

$$
\begin{aligned}
& I_{1}\left(\ddot{\theta}-\dot{\phi}^{2} \sin \theta \cos \theta\right)+I_{3} \dot{\phi}(\dot{\psi}+\dot{\phi} \cos \theta) \sin \theta+\lambda \dot{\theta}+V_{\theta} \\
& =\Gamma_{\theta}, \\
& I_{1}\left(\ddot{\phi} \sin ^{2} \theta+2 \dot{\phi} \dot{\theta} \sin \theta \cos \theta\right)+I_{3} \cos \theta \frac{d}{d t}(\dot{\psi}+\dot{\phi} \cos \theta) \\
& \quad-I_{3}(\dot{\psi}+\dot{\phi} \cos \theta) \dot{\theta} \sin \theta+\lambda \dot{\phi} \sin ^{2} \theta+V_{\phi}=\Gamma_{\phi},
\end{aligned}
$$

$$
I_{3} \frac{d}{d t}(\dot{\psi}+\dot{\phi} \cos \theta)+\lambda^{\prime}(\dot{\psi}+\dot{\phi} \cos \theta)=\Gamma_{\psi},
$$

where $V_{\theta}=\partial V / \partial \theta$ and $V_{\phi}=\partial V / \partial \phi$. The expression $(\dot{\psi}$ $+\dot{\phi} \cos \theta$ ) was left unbroken wherever it appears in the above equations because it represents the component of the angular velocity vector $\boldsymbol{\omega}$ along the symmetry axis, and we make use of this fact in the interpretation of the dissipative torques in terms of the components of $\boldsymbol{\omega}$, as follows.

Let us define the following four unit vectors: $\mathbf{z}$, along the laboratory $z$ axis; c, along the particle's symmetry axis; a, perpendicular to the plane containing $\mathbf{c}$ and $\mathbf{z}$ ( $\widehat{c z}$ plane); and b, perpendicular to the $\widehat{c a}$ plane:

$$
\mathbf{z}=(0,0,1)
$$

$$
\mathbf{c}=(\sin \theta \cos \phi, \sin \theta \sin \phi, \cos \theta),
$$

$$
\begin{gathered}
\mathbf{a}=\frac{\mathbf{z} \times \mathbf{c}}{\sin \theta}=(-\sin \phi, \cos \phi, 0), \\
\mathbf{b}=\mathbf{c} \times \mathbf{a}=(-\cos \theta \cos \phi,-\cos \theta \sin \phi, \sin \theta) .
\end{gathered}
$$

As a notation to be used throughout this work, subscripts $z, c, a$, or $b$ on a vector indicate its orthogonal projection on the $\mathbf{z}, \mathbf{c}, \mathbf{a}$, or $\mathbf{b}$ directions, and subscript $\bar{c}$ indicates the vector's projection on the plane perpendicular to $\mathbf{c}$.

The particle's angular velocity vector $\boldsymbol{\omega}$ may be decomposed into a sum of two vectors, perpendicular and parallel to $c$, respectively,

$$
\boldsymbol{\omega}=\boldsymbol{\omega}_{\bar{c}}+\omega_{c} \mathbf{c}
$$

with

$$
\begin{aligned}
\boldsymbol{\omega}_{\bar{c}}= & \mathbf{c} \times \dot{c} \\
= & (-\dot{\theta} \sin \phi-\dot{\phi} \sin \theta \cos \theta \cos \phi, \dot{\theta} \cos \phi \\
& \left.-\dot{\phi} \sin \theta \cos \theta \sin \phi, \dot{\phi} \sin ^{2} \theta\right)
\end{aligned}
$$

and

$$
\omega_{c}=\dot{\psi}+\dot{\phi} \cos \theta
$$

The orthogonal projection of $\boldsymbol{\omega}_{\bar{c}}^{-}$on the $z$ axis is

$$
\omega_{\overline{c z}}=\boldsymbol{\omega}_{\bar{c}} \cdot \mathbf{z}=\dot{\phi} \sin ^{2} \theta,
$$

and the orthogonal projection of $\boldsymbol{\omega}$ (or of $\boldsymbol{\omega}_{\bar{c}}$ ) on the direction perpendicular to the $\widehat{\mathbf{c z}}$ plane is

$$
\omega_{a}=\boldsymbol{\omega} \cdot \mathbf{a}=\boldsymbol{\omega}_{\bar{c}} \cdot \mathbf{a}=\dot{\theta} .
$$

Thus we see that the dissipative torques present in Eqs. (5a), $(5 b)$, and $(5 \mathrm{c})$ are given by $\omega_{a}, \omega_{\bar{c} z}$, and $\omega_{c}$, respectively, times the dissipation parameters $\lambda$ or $\lambda^{\prime}$.

The noise torques will be treated along these same lines. We start by defining the noise torque vector by its orthogonal components: 


$$
\boldsymbol{\Gamma}=\Gamma_{a} \mathbf{a}+\Gamma_{b} \mathbf{b}+\Gamma_{c} \mathbf{c} .
$$

The noise becomes completely defined by stating the statistics of its three components. The usual procedure is to consider them as statistically independent, Gaussian white noise. This is, however, not a necessary assumption and we leave it open for future modeling. What we need now is to know how the three components come into Eqs. (5). Guided by the above decomposition of the dissipative torque, we are led to identify

$$
\begin{gathered}
\Gamma_{\theta}=\Gamma_{a}, \\
\Gamma_{\phi}=\Gamma_{\bar{c} z}=\Gamma_{\bar{c}} \cdot \mathbf{z}=\Gamma_{b} \sin \theta, \\
\Gamma_{\psi}=\Gamma_{c} .
\end{gathered}
$$

Before we proceed to deduce the equations of motion for the general case of magnetic particles in suspensions we show, in Sec. III, how to obtain, from Eqs. (5), the equations of motion for the spherical coordinates of a monodomain magnetic moment.

\section{EQUATIONS OF MOTION FOR A MAGNETIC MOMENT}

The magnetic moment $\boldsymbol{\mu}$ of a monodomain particle is related to its internal angular momentum $\mathbf{S}$ by $\boldsymbol{\mu}=\gamma \mathbf{S}$, where $\gamma$ is the gyromagnetic factor. Although the modulus $S$ of $\mathbf{S}$ is taken as constant in most works on superparamagnetism and magnetic fluids, for very small particles its oscillation may be significant and we prefer to allow it to be time dependent. The modern technology allows the preparation of samples with magnetic particles whose diameters are smaller than 20 $\AA$ [17], and superparamagnetic clusters containing only 12 magnetic atoms were also reported [18]. We can model the magnetic moment by a rotating charged particle, in the limit of zero moments of inertia, $I_{1} \rightarrow 0, I_{3} \rightarrow 0$, and $\dot{\psi} \rightarrow \infty$, so that $I_{3} \dot{\psi}=S$. Because in Sec. IV we will work with the joint system, a particle and its fluctuating magnetic moment, we write the generalized coordinates, potential energy, dissipative and noise torques, with a notation distinct from that corresponding to the particle. That is, we make the following substitutions: $\theta \rightarrow \vartheta, \quad \phi \rightarrow \varphi, I_{3} \dot{\psi} \rightarrow S, \quad V \rightarrow W, \lambda \rightarrow \xi, \lambda^{\prime}$ $\rightarrow \xi^{\prime}$, and $\Gamma \rightarrow \mathcal{T}$. We also introduce two modifications in the equation corresponding to Eq. (5c), namely, we write $S$ $-S_{0}$ instead of $S$ in the dissipative term, and introduce a torque $W_{s}$, whose origin will be explained below. In the stated limit and with this new notation, the system of equations (5) becomes

$$
\begin{gathered}
S \dot{\varphi} \sin \vartheta+\xi \dot{\vartheta}+W_{\vartheta}=\mathcal{T}_{\vartheta}, \\
\dot{S} \cos \vartheta-S \dot{\vartheta} \sin \vartheta+\xi \dot{\vartheta} \sin ^{2} \vartheta+W_{\varphi}=\mathcal{T}_{\varphi}, \\
\dot{S}+\xi^{\prime}\left(S-S_{0}\right)+W_{s}=\mathcal{T}_{s} .
\end{gathered}
$$

Here we have written $S-S_{0}$, instead of $S$, in the dissipation term of Eq. (7c) to account for the fact that the relaxation of the fluctuations of $S$ is towards a most probable (equilibrium) value $S_{0}$, and not toward 0 . It may appear strange that, even though we have derived the equations of motion for $\mathbf{S}$ from the equations of motion for a symmetric particle, in a convenient limit we now have to add a term "ad hoc" $\left(S_{0}\right)$, which does not have an equivalent in the particle's equations. This is so because in classical physics the equilibrium magnetization is always zero. Nonzero equilibrium magnetic moments can only exist because of the quantum mechanical nature of matter and, therefore, cannot be deduced from a pure classical approach. The torque $W_{s}$ was introduced because a crystal field may have an effective interaction with $\boldsymbol{\mu}$, with origin in an orbital contribution to $\mathbf{S}$ [19], with a possible torque component parallel to $\mathbf{S}$. There is not an equivalent term in Eq. (5c) because of the assumed axial symmetry of the particle.

It is interesting to study the behavior of Eqs. (7) in the absence of noise, $\mathcal{T}_{i}=0$, and with $W_{s}=0$. Equation (7c) has then the trivial stationary solution $S=S_{0}$. Assuming this constant value for $S$ in Eqs. (7a) and (7b), they reduce to

$$
\begin{gathered}
S_{0} \dot{\varphi} \sin \vartheta+\xi \dot{\vartheta}+W_{\vartheta}=0, \\
-S_{0} \vartheta \sin \vartheta+\xi \dot{\varphi} \sin ^{2} \vartheta+W_{\varphi}=0 .
\end{gathered}
$$

The conservative torques $-W_{\vartheta}$ and $-W_{\varphi}$ usually receive contributions from two different origins, the interaction of $\mathbf{S}$ with a crystalline, anisotropy field and/or with a magnetic field, which can also be of several different origins. In the case of magnetic field $\mathbf{H}$, the potential energy is $W=-\boldsymbol{\mu}$ - H. With a little algebraic work one can show, in this case, that the set of equations (8) is equivalent to the well known Gilbert's equation [10],

$$
\frac{d \boldsymbol{\mu}}{d t}=\gamma \boldsymbol{\mu} \times\left[\mathbf{H}-\frac{\xi}{\mu^{2}} \frac{d \boldsymbol{\mu}}{d t}\right]
$$

for $\boldsymbol{\mu}=\gamma \mathbf{S}$ and $S=S_{0}$. This equation was used by Brown [12] as a starting point for his stochastic theory of superparamagnetism, where he assumed the magnetic field $\mathbf{H}$ to contain a noise term. A more general theory for superparamagnetism, which also allows for oscillations on the modulus $\mu=\gamma S$ of the magnetic moment, was worked out by Ricci and Scherer [20,21,15], based on the set of equations (7). For this reason we will not continue to explore the consequences of Eqs. (7) in the present paper, turning, instead, to the more general approach, where the rotation of the mechanical particle is taken into account, in addition to the motion of $\mathbf{S}$ relative to the particle.

\section{EQUATIONS OF MOTION FOR A SMALL MAGNETIC PARTICLE IN SUSPENSION}

In recent years several researchers $[1,4,14,22]$ drew attention to the importance of the motion of the magnetic particle, its inertia and viscous interaction with the fluid, to the dynamic magnetic susceptibility of ferrofluids. A theoretical treatment of this problem, which is both more fundamental and more general than those previously published, follows naturally from the context described above. 
Taken together, the systems of equations (5) and (7) contain all the degrees of freedom relevant to the problem. To the potential energy terms, $V$ in Eqs. (5) and $W$ in Eqs. (7), the interaction energy between the magnetic moment and the particle, which we will denote by $U$, has to be added. Due to the particle's symmetry, this term can only depend on $S$, and on the angle between $\mathbf{S}$ and the symmetry axis $\mathbf{c}$. It is convenient to define another orthogonal set of unit vectors, related to the direction of the magnetic moment, namely, $\mathbf{s}$, in the $\mathbf{S}$ direction, $\mathbf{u}$, perpendicular to the $\widehat{\mathbf{s z}}$ plane, and $\mathbf{v}$, perpendicular to the $\widehat{\text { su }}$ plane:

$$
\begin{gathered}
\mathbf{s}=\frac{\mathbf{S}}{S}=(\sin \vartheta \cos \varphi, \sin \vartheta \sin \varphi, \cos \vartheta), \\
\mathbf{u}=\frac{\mathbf{z} \times \mathbf{s}}{\sin \vartheta}=(-\sin \varphi, \cos \varphi, 0), \\
\mathbf{v}=\mathbf{s} \times u=(-\cos \vartheta \cos \varphi,-\cos \vartheta \sin \varphi, \sin \vartheta) .
\end{gathered}
$$

The interaction energy $U$ can then be written as $U(S, \mathbf{s} \cdot \mathbf{c})$. In principle, the particle can interact also with other fields, besides $\mathbf{H}$, as is the case if it has an electric dipole and an electric field is present. For this reason we also keep the potential energy $V(\theta, \phi)$ in the new set of equations.

The dissipative interaction associated with the rotation of $\mathbf{S}$ relative to the particle will be written in terms of the relative angular velocity vector. Since only rotations perpendicular to $\mathbf{S}$ can lead to a meaningful interaction torque with origin on the relative motion, we define the relative angular velocity $\boldsymbol{\omega}_{r}$ as

$$
\boldsymbol{\omega}_{r}=\varpi-\boldsymbol{\omega}_{\bar{s}},
$$

where

$$
\varpi=\mathbf{s} \times \dot{\mathbf{s}}
$$

is the angular velocity of rotation of the magnetic moment with respect to the laboratory, and

$$
\boldsymbol{\omega}_{\bar{s}}^{-}=\mathbf{s} \times \boldsymbol{\omega} \times \mathbf{s}=\boldsymbol{\omega}-(\mathbf{s} \cdot \boldsymbol{\omega}) \mathbf{s}
$$

is the orthogonal projection of the particle's angular velocity $\boldsymbol{\omega}$ on the plane perpendicular to $\mathbf{S}$. The dissipative interaction torque on the particle is then $+\xi \boldsymbol{\xi} \boldsymbol{\omega}_{r}$. The plus sign is because of the way we defined $\boldsymbol{\omega}_{r}$, where the particle's angular velocity appears with a minus sign. Guided by the interpretation of the dissipative torque terms of Eqs. (5) in terms of angular velocity components, as explained below the aforementioned equations, we immediately write the dissipative torque terms to be added to the left-hand sides (therefore, with a minus sign) of Eqs. (5), namely,

$$
\begin{gathered}
-\xi \omega_{r a}=-\xi \boldsymbol{\omega}_{\mathbf{r}} \cdot \mathbf{a}, \\
-\xi \omega_{r \bar{c} z}=-\xi\left[\boldsymbol{\omega}_{\mathbf{r}}-\left(\boldsymbol{\omega}_{\mathbf{r}} \cdot \mathbf{c}\right) \mathbf{c}\right] \cdot \mathbf{z}=-\xi\left(\omega_{r z}-\omega_{r c} \cos \theta,\right. \\
-\xi \omega_{r c}=-\xi \boldsymbol{\omega}_{\mathbf{r}} \cdot \mathbf{c} .
\end{gathered}
$$

Of course, all these scalar products, as well as those which follow in the next equations, may be easily written as functions of the four angles $\theta, \phi, \vartheta$, and $\varphi$, and their time derivatives, by using Eqs. (6) and (10). However, because scalar products are very easily handled in numerical procedures, we prefer to leave them in this form.

Clearly, the torque on the magnetic moment, due to the relative motion, is the "reaction" to the torque on the particle, i.e., it is equal to $-\xi \boldsymbol{\omega}_{\mathrm{r}}$, and, in place of $\xi \dot{\vartheta}$ and $\xi \dot{\varphi} \sin ^{2} \vartheta$ in Eqs. (7) we shall use (remembering that $\boldsymbol{\omega}_{r \bar{s}}$ $\left.=\omega_{r}\right)$

$$
\begin{gathered}
\xi \omega_{r u}=\xi \boldsymbol{\omega}_{\mathbf{r}} \cdot \mathbf{u}, \\
\xi \omega_{r z}=\xi \boldsymbol{\omega}_{\mathbf{r}} \cdot \mathbf{z} .
\end{gathered}
$$

No term coming from the relative angular velocity $\boldsymbol{\omega}_{\mathbf{r}}$ has to be added to Eq. (7c), because $\boldsymbol{\omega}_{\mathbf{r}}$ is perpendicular to $\mathbf{S}$. However, there is a term $\xi^{\prime}\left(S-S_{0}\right)$ already present in that equation, with its origin in the (quantum) fluctuations of $S$, and this term will be kept. Since angular momentum has to be conserved, its reaction counterpart on the particle has to be added to Eqs. (5). Calling

$$
\mathcal{R}=\left(S-S_{0}\right) \mathbf{s},
$$

the terms to be added to the left-hand sides of Eqs. (5) are

$$
\begin{aligned}
-\xi^{\prime} \mathcal{R}_{a} & =-\xi^{\prime} \mathcal{R} \cdot \mathbf{a}=-\xi^{\prime}\left(S-S_{0}\right) \mathbf{s} \cdot \mathbf{a}, \\
-\xi^{\prime} \mathcal{R}_{\bar{c} z} & =-\xi^{\prime}[\mathcal{R}-(\mathcal{R} \cdot \mathbf{c}) \mathbf{c}] \cdot z \\
& =-\xi^{\prime}\left(S-S_{0}\right)[s-(s \cdot c) c] \cdot z, \\
-\xi^{\prime} \mathcal{R}_{c} & =-\xi^{\prime} \mathcal{R} \cdot \mathbf{c}=-\xi^{\prime}\left(S-S_{0}\right) \mathbf{s} \cdot \mathbf{c} .
\end{aligned}
$$

The noise torques of interaction between the particle and the magnetic moment can be written down along the same lines of procedure as used for the noise torques of the fluid on the particle at the end of Sec. II. We assume three orthogonal, independent, noise torque vectors, along the unit vectors defined with respect to the direction of the magnetic moment:

$$
\mathcal{T}=\mathcal{T}_{s} \mathbf{s}+\mathcal{T}_{u} \mathbf{u}+\mathcal{T}_{v} \mathbf{v}
$$

$\mathcal{T}$ being the torque on the magnetic moment, then the torque on the particle is $-\mathcal{T}$. Following the same line of reasoning as above, we identify the torques in Eqs. (7):

$$
\begin{aligned}
\mathcal{T}_{\vartheta} & =\mathcal{T}_{u}, \\
\mathcal{T}_{\varphi}=\mathcal{T}_{\bar{s} z} & =\mathcal{T}_{v} \sin \vartheta, \\
\mathcal{T}_{s} & =\mathcal{T}_{s} .
\end{aligned}
$$

Correspondingly, the following terms have to be added to the right-hand sides of Eqs. (5):

$$
\begin{gathered}
\mathcal{T}_{\theta}=-\mathcal{T}_{a}=-\mathcal{T} \cdot \mathbf{a}, \\
\mathcal{T}_{\phi}=-\mathcal{T}_{\bar{c} z}=-[\mathcal{T}-(\mathcal{T} \cdot \mathbf{c}) \mathbf{c}] \cdot \mathbf{z},
\end{gathered}
$$




$$
\mathcal{T}_{\psi}=-\mathcal{T}_{c}=-\mathcal{T} \cdot \mathbf{c} .
$$

Therefore, the state of the composed system, the particle and its magnetic moment, is described by the six generalized coordinates $\theta, \phi, \psi, \vartheta, \varphi$, and $S$, whose dynamical behavior is governed by the following set of coupled differential equations:

$$
\begin{aligned}
I_{1}\left(\ddot{\theta}-\dot{\phi}^{2} \sin \theta \cos \theta\right)+I_{3} \dot{\phi}(\dot{\psi}+\phi \cos \theta) \sin \theta+\lambda \dot{\theta}-\xi \omega_{r a} \\
-\xi^{\prime} \mathcal{R}_{a}+V_{\theta}+U_{\theta}=\Gamma_{a}-\mathcal{T}_{a},
\end{aligned}
$$

$$
I_{1}\left(\ddot{\phi} \sin ^{2} \theta+2 \dot{\phi} \dot{\theta} \sin \theta \cos \theta\right)+I_{3} \cos \theta \frac{d}{d t}(\dot{\psi}+\dot{\phi} \cos \theta)
$$$$
-I_{3}(\dot{\psi}+\phi \cos \theta) \dot{\theta} \sin \theta+\lambda \dot{\phi} \sin ^{2} \theta-\xi \omega_{r \bar{c} z}-\xi^{\prime} \mathcal{R}_{\bar{c} z}
$$$$
+V_{\phi}+U_{\phi}=\Gamma_{b} \sin \theta-\mathcal{T}_{\bar{c} z},
$$

$$
\begin{aligned}
& I_{3} \frac{d}{d t}(\dot{\psi}+\dot{\phi} \cos \theta)+\lambda^{\prime}(\dot{\psi}+\dot{\phi} \cos \theta)-\xi \omega_{r c}-\xi^{\prime} \mathcal{R}_{c} \\
& \quad=\Gamma_{c}-\mathcal{T}_{c},
\end{aligned}
$$

$$
S \dot{\varphi} \sin \vartheta+\xi \omega_{r u}+W_{\vartheta}+U_{\vartheta}=+\mathcal{T}_{u},
$$

$\dot{S} \cos \vartheta-S \dot{\vartheta} \sin \vartheta+\xi \omega_{r \bar{c} z}+W_{\varphi}+U_{\varphi}=+\mathcal{T}_{\bar{s} z}$,

$$
\dot{S}+\xi^{\prime}\left(S-S_{0}\right)+U_{S}=\mathcal{T}_{s} .
$$

This set of six equations is of very general applicability in magnetic suspensions. It allows for a large variety of modeling: There are three independent conservative interaction potentials $V, U$ and $W$, four dissipative parameters $\lambda, \lambda^{\prime}, \xi$, and $\xi^{\prime}$, and also the noise torques $\Gamma$ and $\mathcal{T}$, whose statistical properties are open for modeling. Particle-particle interaction was not explicitly taken into account.

\section{DYNAMIC SUSCEPTIBILITY}

To calculate, from the set of equations (12), the dynamic magnetic susceptibility, and therefore the absorption lines of magnetic resonance, it is better to transform them into the typical form of first order differential Langevin equations [23]. Noting that the first three equations are second order, we introduce new variables

$$
\begin{gathered}
\dot{\theta}=\eta, \\
\dot{\phi}=\nu, \\
\dot{\psi}+\dot{\phi} \cos \theta=\rho,
\end{gathered}
$$

and transform Eqs. (12), so that, together with Eqs. (13), we have a set of nine first order equations. For example, Eq. (12a) becomes

$$
\begin{aligned}
& I_{1}\left(\dot{\eta}-\nu^{2} \sin \theta \cos \theta\right)+I_{3} \nu \rho \sin \theta+\lambda \eta-\xi \omega_{r a}-\xi^{\prime} \mathcal{R}_{a}+V_{\theta} \\
& \quad+U_{\theta}=\Gamma_{a}-\mathcal{T}_{a},
\end{aligned}
$$

We use also the Wiener processes $W_{j}(t)$, which are related to the white noise components $\Gamma_{j}$ [or whatever appears at the right-hand side of Eqs. (12)] by

$$
W_{j}(t)=\int_{0}^{t} \Gamma_{j}\left(t^{\prime}\right) d t^{\prime},
$$

and make the usual substitutions $\Gamma_{j}(t) d t \rightarrow d W_{j}(t)$ to write the set of stochastic differential equations in the form

$$
d X_{i}(t)=A_{i}[\mathbf{X}(t), t] d t+\sum_{j} B_{i j}[\mathbf{X}(t)] d W_{j}(t),
$$

where $X_{i}(t)$ are the dependent variables $\eta, \nu, \rho, \theta, \phi, \psi, \vartheta$, $\varphi$, and $S$, and $A_{i}[\mathbf{X}(t), t]$ and $B_{i j}[\mathbf{X}(t)]$ are obtained by comparison between Eq. (14) and those from the set of first order equations mentioned above, after the expressions for $V, U$, and $W$ are introduced. In the typical case of magnetic resonance, with a strong constant magnetic field $\mathbf{H}_{0}$ parallel to the $\mathbf{z}$ axis and a periodic weak field $\mathbf{F}(t)$ perpendicular to it, $A_{i}[\mathbf{X}(t), t]$ turn out to be written in the form

$$
A_{i}[\mathbf{X}(t), t]=\sum_{j} \gamma_{i j}(\mathbf{X}) F_{j}(t)+A_{i}^{0}(X) .
$$

Following the procedure of Ref. [15], the response functions are then given by

$$
\Phi_{i j}(t)=\sum_{k}\left\langle\gamma_{k j}(\mathbf{x}) \partial_{k}\left\langle X_{i}(t \mid \mathbf{x})\right\rangle_{0}\right\rangle_{\mathrm{eq}}
$$

The symbol $X_{i}(t \mid \mathbf{x})$ refers to the stochastic variable $X_{i}$ at time $t$, given that the "vector" of stochastic variables $\mathbf{X}$ had a value $\mathbf{x}$ at an initial time $t=0 ;\left\langle X_{i}(t \mid \mathbf{x})\right\rangle_{0}$ is an average over many realizations of $X_{i}$, from 0 to $t$, in the absence of the perturbing field $\mathbf{F}(t)$, starting from the point $\mathbf{x}, \partial_{k}$ means derivative with respect to the $k$ component of the initial "point" $\mathbf{x}$; and $\langle\cdots\rangle_{\mathrm{eq}}$ is an average over the equilibrium distribution of initial points. This equilibrium average may include an average over the distribution of the particle's characteristics, if polydispersity is to be considered. For example, if the particles are all made of the same material and have the same shape, varying only in size, assuming some given distribution of a linear dimension $r$, then the other particle's parameters shall be scaled accordingly. For example,

$$
S_{0} \propto r^{3}, \quad I \propto r^{5}, \quad \lambda \propto r^{3} .
$$

As shown in Ref. [15], Eq. (16) may be evaluated from numerical simulations of Eq. (14). From the results obtained for $\Phi_{i j}(t)$, we can then calculate the susceptibility $\chi_{i j}(\omega)$ by numerical Fourier-Laplace transform.

\section{SOME LIMIT CASES}

Several interesting limit situations are readily obtained from Eqs. (12). The "superparamagnetic" limit, for which the particle's coordinates $\theta, \phi$, and $\psi$, are taken as constants, so that the system reduces to the last three equations [or, 
equivalently, to Eqs. (7)], was treated in three previous papers by Ricci and Scherer [20,21,15]. A further simplification, in this limit, which is appropriate for most cases of practical interest, follows by assuming $S=S_{0}=$ const. In this case the only relevant equations are Eqs. (12d) and (12e), and, moreover, the term in $\dot{S}$ also vanishes. The noise torques $\mathcal{T}_{u}$ and $\mathcal{T}_{\bar{s} z}$ may then be written in terms of a stochastic magnetic field, rendering our set of equations in a form equivalent to Brown's generalization [12] of Gilbert's equation [Eq. (9)]. This case was treated by several authors, and a very interesting account was given in a recent paper by GarciaPalacios and Lázaro [24], where much numerical work was presented.

The "blocked" limit (also called the "Brownian" limit [25] or "inertial limit" [2]), corresponds to the case when the magnetic moment is blocked along the particle's symmetry direction, i.e., $\vartheta=\theta$ and $\varphi=\phi$. This may happen because the sample is kept below the "blocking temperature" $T_{B}$ [26], or because the material is so highly anisotropic that the magnetic moments only exists parallel to the easy axis [18]. The particle is still immersed in a fluid carrier, being able to rotate, together with its magnetic moment.

In terms of the set of equations (12), the blocked limit is obtained by assuming an interaction potential $U$ of the form $-U_{0} \delta(\mathbf{s}-\mathbf{c})$, with $U_{0} \rightarrow \infty$, so that the only states energetically possible are those with $\mathbf{s}=\mathbf{c}$, i.e., $\vartheta=\theta$ and $\varphi=\phi$. By summing Eq. (12a) with Eq. (12d) and Eq. (12b) with Eq. (12e) the interaction terms $U_{\theta}$ and $U_{\vartheta}$ as well as $U_{\phi}$ and $U_{\varphi}$ cancel out. The terms containing $\omega_{r a}, \omega_{r \bar{c} z}, \mathcal{R}_{a}, \mathcal{R}_{\bar{c} z}, \mathcal{T}_{a}$, and $\mathcal{T}_{\bar{c} z}$ become identically zero, and $\mathcal{R}_{c}$ becomes ( $S$ $\left.-S_{0}\right)$. Choosing $\theta$ and $\phi$ to denote the common polar angles, the system of equations, in the notation of the previous section, becomes

$$
\begin{aligned}
& I_{1}\left(\dot{\eta}-\nu^{2} \sin \theta \cos \theta\right)+I_{3} \nu \rho \sin \theta+\lambda \eta+V_{\theta}+S \nu \sin \theta+W_{\theta} \\
& =\Gamma_{a}, \\
& I_{1}\left(\dot{\nu} \sin ^{2} \theta+2 \nu \eta \sin \theta \cos \theta\right)+I_{3} \cos \theta \dot{\rho}-I_{3} \rho \eta \sin \theta \\
& \quad+\lambda \nu \sin ^{2} \theta+V_{\phi}+S \cos \theta-S \eta \sin \theta+W_{\phi}=\Gamma_{b} \sin \theta,
\end{aligned}
$$

$$
\begin{gathered}
I_{3} \dot{\rho}+\lambda^{\prime} \rho-\xi^{\prime}\left(S-S_{0}\right)=\Gamma_{c}-\mathcal{T}_{c}, \\
\dot{S}+\xi^{\prime}\left(S-S_{0}\right)=+\mathcal{T}_{c} .
\end{gathered}
$$

This is still a rather general set of equations. A first obvious simplification occurs, in most cases of interest, when $S$ $=S_{0}$. Then also $\dot{S}=0$ and $\mathcal{T}_{c}=0$, and the system is reduced to three equations. Much work has been done in this case, mainly in the context of electric dipolar molecules, for which $S=S_{0}=0$. For example, McConnell [27], Coffey, Kalmikov Waldron [28], and Gaiduk and McConnell [29] describeddielectric relaxation and dynamics of polar molecules in great detail.

As a simple illustration we will assume a constant modulus for the magnetic moment, i.e., $S=S_{0}$, and for the interaction potential we consider only $W=-\boldsymbol{\mu} \cdot \mathbf{H}=-\gamma S_{0} \mathbf{s} \cdot \mathbf{H}$,

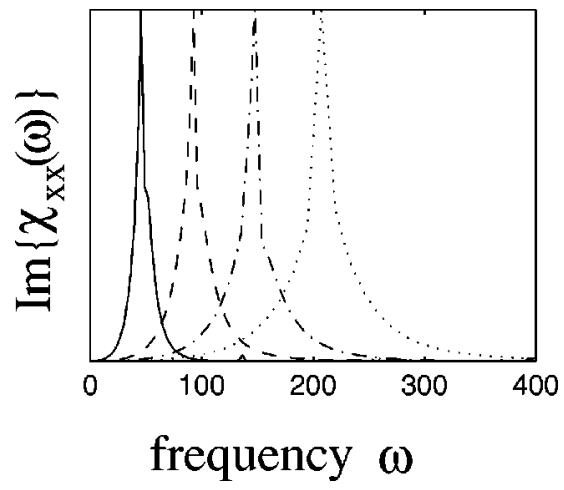

FIG. 1. Imaginary part of the suceptibility for different values of the moment of inercia $I_{1}$. Full line: $I_{1}=1.0$; dashed line: $I_{1}$ $=0.25$; dot-dashed line: $I_{1}=0.10$; dotted line: $I_{1}=0.05$.

where $\mathbf{H}=H_{0} \mathbf{z}$ is a constant field. The interaction energy between the magnetic moment and the field is then

$$
W=-\boldsymbol{\mu} \cdot \mathbf{H}=-S_{0} \gamma H_{0} \cos \theta .
$$

With these simplifications, the system of equations (18) becomes

$$
\begin{gathered}
I_{1}\left(\dot{\eta}-\nu^{2} \sin \theta \cos \theta\right)+I_{3} \nu \rho \sin \theta+\lambda \eta+S_{0} \nu \sin \theta \\
+S_{0} \gamma H_{0} \sin \theta=\Gamma_{a}, \\
I_{1}\left(\dot{\nu} \sin ^{2} \theta+2 \nu \eta \cos \theta\right)+I_{3} \cos \theta \dot{\rho}-I_{3} \rho \eta \sin \theta+\lambda \nu \sin ^{2} \theta \\
-S_{0} \eta \sin \theta=\Gamma_{b} \sin \theta \\
I_{3} \dot{\rho}+\lambda^{\prime} \rho=\Gamma_{c} .
\end{gathered}
$$

We will consider in this simple illustration only the limit of very weak noise, $\Gamma \simeq 0$. Then Eq. (19c) has the approximate stationary solution $\rho \simeq 0$. Therefore, we neglect $\rho$ in Eqs. (19a) and (19b), which become

$$
I_{1}\left(\dot{\eta}-\nu^{2} \sin \theta\right)+\lambda \eta+S_{0} \nu \sin \theta+S_{0} \gamma H_{0} \sin \theta=\Gamma_{a},
$$

$$
I_{1}(\dot{\nu} \sin \theta+2 \nu \eta \cos \theta)+\lambda \nu \sin \theta-S_{0} \eta=\Gamma_{b},
$$

which, together with the definition of $\eta$ and $\nu$ in Eqs. (13), form a set of four first order Langevin equations. It requires simple algebra to show that, in the appropriate limit, i.e., $\rho$ $=\dot{\psi}+\nu \cos \theta \simeq 0$ and mutatis mutandis from our notation to the notation of Ref. [28], Eqs. (20) are the same as Eqs. (10.3.2-a) and (10.3.2b) of the latter paper. We note that for the case of Ref. [28], for a particle with an electric dipole, the terms in $S_{0}$ are zero (there is no angular momentum associated with the electric dipole) and in the interaction term with the external field $S_{0} \gamma H_{0} \rightarrow \mu F$.

Following the procedure outlined in Sec. V, with arbitrarily chosen values for the parameters $S_{0}, \lambda$, and $\gamma H_{0}$, and for four distinct values of the moment of inertia $I_{1}=0.05$, $0.10,0.25$, and 1.0 , we calculated the dynamic susceptibility. The imaginary part $\chi_{x x}^{\prime \prime}(\omega)$ is shown, in arbitrary units, in Fig. 1. We do not claim that the values we used for the 
parameters are realistic for ferrofluids; this simple example only qualitatively shows the effect of the particle's inertia on the magnetic resonance lines.

Another interesting limit is the noninertial limit, $I=0$. If we further simplify our system of equations (12) by assuming $S=S_{0}=$ const, and therefore $U_{S}=\mathcal{T}_{s}=0$, the system becomes a system of five first order equations for the variables $\theta, \phi, \psi, \vartheta$, and $\varphi$, the first three of these being simply equilibrium equations between all torques on the mechanical particle (dissipative and Brownian interaction with the liquid and with the magnetic moment), and the last two becoming equivalent to Brown's generalization of Gilber's equation [12]. This case was extensively studied by Shliomis and Stepanov [3], in the context of their "egg'" model.

\section{ACKNOWLEDGMENTS}

We thank Professor Hans Herrmann, director of ICA1, for the hospitality of his institute. This work was supported in part by $\mathrm{CNPq}$ (Brazil) and by Alexander von Humboldt Foundation (Germany).
[1] Y. L. Raikher and V. V. Rusakov, Phys. Rev. E 54, 3846 (1996).

[2] W. T. Coffey and Yu. P. Kalmykov, J. Magn. Magn. Mater. 164, 133 (1996).

[3] M. I. Shliomis and V. I. Stepanov, Theory of the Dynamic Susceptibility of Magnetic Fluids, in Relaxation Phenomena in Condensed Matter, Advanced Chemistry in Physics Series Vol. 87 (1994) (unpublished).

[4] P. C. Morais, A. L. Tronconi, F. A. Tourinho, and F. Pelegrini, Solid State Commun. 101, 693 (1997).

[5] P. C. Fannin, S. W. Charles, and T. Relihan, J. Magn. Magn. Mater. 162, 319 (1996); See also P. C. Fannin, D. Vincent, G. Massart, P. Perov, and S. Neveu, Eur. Phys. J.: Appl. Phys. 8, 247 (1999); P. C. Fannin, P. A. Perov, and S. W. Charles, J. Phys. D 32, 2367 (1999).

[6] D. Vincent, S. Neveu, L. Jorat, and G. Noyel, J. Magn. Magn. Mater. 163, 216 (1996).

[7] R. V. Upadhyay, D. Srinivas, and R. V. Mehta, J. Magn. Magn. Mater. 214, 105 (2000).

[8] P. C. Fannin, L. Kinsella, and S. W. Charles, J. Phys. D 30, 533 (1997).

[9] K. Raj, B. Moskowitz, and R. Casciari, J. Magn. Magn. Mater. 149, 174 (1995).

[10] T. L. Gilbert, Phys. Rev. 100, 1243 (1955).

[11] L. Landau and E. Lifshitz, Phys. Z. Sowjetunion 8, 153 (1935).

[12] W. F. Brown, Phys. Rev. 130, 1677 (1963).

[13] P. Debye, Polar Molecules (Chemical Catalog, New York, 1929).

[14] P. C. Fannin and W. T. Coffey, Phys. Rev. E 52, 6129 (1995).
[15] T. F. Ricci and C. Scherer, J. Stat. Phys. 86, 803 (1997).

[16] H. Goldstein, Classical Mechanics, 2nd ed. (Addison-Wesley, Reading, MA, 1980).

[17] J. P. Chen, C. M. Sorensen, K. J. Klabunde, and G. C. Hadjipanayis, Phys. Rev. B 51, 11527 (1995).

[18] M. A. Novak, R. Sessoli, A. Caneschi, and D. Gatteschi, J. Magn. Magn. Mater. 146, 211 (1995).

[19] R. M. White, Quantum Theory of Magnetism (Springer, Berlin, 1983).

[20] T. F. Ricci and C. Scherer, Braz. J. Phys. 21, 172 (1991).

[21] T. F. Ricci and C. Scherer, J. Stat. Phys. 67, 1201 (1992).

[22] P. C. Fannin, S. W. Charles, and T. Relihan, J. Phys. D 28, 1765 (1995).

[23] C. W. Gardiner, Handbook of Stochastic Methods (Springer, Berlin, 1989).

[24] J. L. Garcia-Palacios and F. J. Lazaro, Phys. Rev. B 58, 14937 (1998).

[25] M. I. Shliomis, Usp. Fiz. Nauk. 112-114, 427 (1974) [Sov. Phys. Usp. 17, 153 (1974)].

[26] D. D. Awschalom and D. P. DiVincenzo, Phys. Today (4), 43 (1995).

[27] J. R. McConnell, Rotational Brownian Motion and Dielectric Theory (Academic, London, 1980).

[28] W. T. Coffey, Yu. P. Kalmikov, and J. T. Waldron, The Langevin Equation (World Scientific, London, 1998).

[29] V. J. Gaiduk and J. R. McConnell, Dielectric Relaxation and Dynamics of Polar Molecules Series in Contemporary Physics, Vol. 8 (World Scientific, London, 1999). 